\title{
Natural Killer Cells in Human Cancer: From Biological Functions to Clinical Applications
}

\author{
Estrella Mariel Levy, ${ }^{1}$ María Paula Roberti, ${ }^{1}$ and José Mordoh ${ }^{1,2}$ \\ ${ }^{1}$ Centro de Investigaciones Oncológicas, Fundación Cáncer e Instituto Alexander Fleming, Cramer 1180, \\ Ciudad Autónoma de Buenos Aires C1426ANZ, Argentina \\ ${ }^{2}$ IIBBA-CONICET, Fundación Instituto Leloir, Avendia Patricias Argentinas 435, \\ Ciudad Autónoma de Buenos Aires C1405BWE, Argentina \\ Correspondence should be addressed to José Mordoh, jmordoh@leloir.org.ar
}

Received 29 December 2010; Accepted 25 February 2011

Academic Editor: Roberto Biassoni

Copyright (๑) 2011 Estrella Mariel Levy et al. This is an open access article distributed under the Creative Commons Attribution License, which permits unrestricted use, distribution, and reproduction in any medium, provided the original work is properly cited.

\begin{abstract}
Natural killer (NK) cells are central components of the innate immunity. In murine models, it has been shown that NK cells can control both local tumor growth and metastasis due to their ability to exert direct cellular cytotoxicity without prior sensitization and to secrete immunostimulatory cytokines like IFN- $\gamma$. The latter participates in cancer elimination by inhibiting cellular proliferation and angiogenesis, promoting apoptosis, and stimulating the adaptive immune system, and it is instrumental for enhancing Ag processing and presentation. Nevertheless, NK cells display impaired functionality and capability to infiltrate tumors in cancer patients. Also, NK cells are feasible targets of stimulation to participate in immunotherapeutic approaches like antibody-based strategies and adoptive cell transfer. Thus, multiple attempts currently aim to manipulate NK for utilization in the immunotherapy of cancer.
\end{abstract}

\section{Introduction}

NK cells are effector lymphocytes of the innate immune system that control several types of tumors by limiting their growth and dissemination [1]. In vitro studies using cells from humans and several other mammalian species, as well as in vivo studies in mice and rats, have long provided evidence that tumor cells are recognized as NK cell targets [2]. There are numerous studies in mice supporting the notion that NK cells are involved in the elimination of tumor cells. The in vivo analyses relied on antibody-mediated depletion of NK cells in mice, targeting either NK1.1 or the glycolipid asialo-GM1 [3-5]. However, NK cell depletion with antibodies to NK1.1 may also affect populations of invariant natural killer $\mathrm{T}$ cells and other NK1.1 ${ }^{+}$T-cell populations. The selectivity of NK cell depletion with asialoGM1 antibodies (Abs) has also been hampered by the expression of asialo-GM1 by several cell types including myeloid cells, epithelial cells, and T-cell subsets. Caution is therefore required when interpreting studies based upon $\mathrm{Ab}$ depletion because of the lack of specificity of $\mathrm{Ab}$ treatment against NK populations. Nevertheless, many other independent studies advocate a role for NK cells in the control of tumor development in mice. Particularly, mouse NK cells are involved in the in vivo rejection of several transplanted tumors, in a manner dependent upon the presence or absence of NK cell receptor ligands expressed by the tumor [6]. More informative studies regarding tumor immunosurveillance are the experiments addressing the control of newly arising tumors. In this respect Schreiber's group demonstrated that frequencies of spontaneously arising tumors or induced by methylcholantrene (MCA) were higher in mice deficient for key effector molecules of NK cells or the respective receptors $[7,8]$. Nevertheless, in a further study from the same group, it was shown that although NK cells are important in the early elimination of MCA-induced tumors, control of the "dormant" tumor state depends mainly on adaptive immunity [9]. Other studies provided evidence that NK cells can recognize and eliminate aberrant cells. The lack of MHC class I expression [10] or the upregulation of NKG2D ligands $[11,12]$ can render tumor cells susceptible to NK cell-mediated lysis. In some of 
these experimental models, NK cell-mediated elimination of tumor cells induced the subsequent development of tumorspecific T-cell responses to the parental tumor cells $[11,13]$ as a bridge between innate and adaptive immune responses [14]. A role for NK cells in tumor immunosurveillance has also been implicated in controlling the growth of Bcell lymphomas that spontaneously arise in mice lacking both perforin and $\beta 2$-microglobulin [15]. Moreover, the blocking of NK cell MHC class-I-specific inhibitory receptors increases NK cell effector function against tumor cells in mice [16]. In addition to their endogenous protective role in tumor models, NK cells are also mediators of the antitumor effects of several recombinant cytokines, such as IL-2, IL12, IL-18, and IL-21 [6]. So far, these studies in vitro and in animal models show the role of NK cells in cancer immunosurveillance.

Clinical and experimental evidence demonstrate that this important role of NK cells holds true in humans. Although the paucity of NK cell-selective deficiencies has limited the characterization of NK cell biological function in vivo in general and in antitumor immunosurveillance in particular [17], there is an 11-year follow-up epidemiologic survey which has shown that the extent of NK cell activity in peripheral blood is associated with cancer risk in adults: low NK cell activity is associated with increased cancer risk [18]. Furthermore, intratumoral NK cell studies have been hampered because of the low numbers of these cells and the difficulty to obtain them from tumor samples. However, in the last few years, novel studies have revealed the phenotypic status and functionality of NK cells in tumor site and also in peripheral blood (PB) of cancer patients. First studies about tumor immune infiltration have shown that the presence of NK cells represents a positive prognostic marker in different carcinomas [19-21]. Further studies in established human tumors showed that there are often only a few infiltrating NK cells which are unlikely to greatly contribute to the elimination of tumor cells [22, 23]. It has been suggested that despite of low NK cell numbers in tumors, due to their inefficient homing into malignant tissues, this situation may be overcome by cytokine-mediated activation in immunotherapeutical regimens [24]. In this respect, all this evidence about NK importance in tumor immune control enhances the appeal of NK cell-based immunotherapeutic approaches. For instance, the beneficial role of NK cells in controlling human malignancies stems from clinical studies of leukemia patients who received alloreactive NK cells in the course of allogeneic hematopoietic stem cell transplantation $[25,26]$. Likewise, several studies have established the importance of $\mathrm{Fc}-\mathrm{Fc} \gamma \mathrm{R}$ interactions for the in vivo antitumor effects of certain monoclonal antibodies [27-30]. In the present work we will revise key aspects of NK cells role from molecular and cellular characteristics to therapeutic applications in cancer patients.

\section{NK Cells in Cancer Patients}

2.1. NK Cells Tumor Infiltration. Primary tumor growth is a complex process, involving many interactions between the tumors and surrounding tissue. A developing tumor influences and is influenced by its stroma, initiates angiogenesis, and interacts with both the adaptive and innate immune systems. The clinicopathological significance of the tumor-infiltrating lymphocytes (TILs) in various human cancers has been an issue of great interest. $\mathrm{CD}^{+}$cytotoxic $\mathrm{T}$ cells (CTL) and NK cells are the most likely effectors for an effective antitumor immunity [31]. Several studies have shown that the infiltration of lymphocytes significantly correlates with a prolonged survival time of patients, at least in certain types of cancer [32-34]. Typically, NK cells are not found in large numbers in advanced human neoplasms, indicating that they do not normally home efficiently to malignant tissues. For instance, a low prevalence of gastric and colorectal (CRC) tumor-infiltrating $\mathrm{CD} 56^{+}$cells in livers with multiple metastases was detected, whereas in cases with solitary metastases a higher degree of lymphocyte infiltration was observed. Moreover, the percentage of intrahepatic NK $\left(\mathrm{CD} 56^{+}\right)$cells was also decreased in patients with metastases compared to those without, being almost twice lower than $\mathrm{CD}^{+}$and $\mathrm{CD}^{+}$. This suggests that low NK cell number could be a reason for the escape of metastatic cells from the mechanisms of liver immune control [35]. In human nonsmall cell lung cancers (NSCLC) NK cells percentage within $\mathrm{CD} 45^{+}$mononuclear cells (MCs) isolated from lung tumors was consistently lower than in $\mathrm{MC}$ from the $\mathrm{PB}$ counterpart and was comparable to that in MC from peritumoral lung tissue. With regard to their localization, NK cells were found in tumor stroma, whereas they were not in direct contact with cancer cells [36]. Another study in NSCLC showed that NK cell infiltration was strikingly heterogenous. Malignant and nonmalignant tissue areas in NSCLC were selectively infiltrated by certain immune cell types with NK cells being displaced from the tumor tissue and displaying low cytotoxic activity [23]. In another study, the $\mathrm{CD} 56^{+} / \mathrm{CD} 16^{+}$cell ratio in renal cell carcinoma microenvironment was found generally lower than 1, suggesting that a predominant number of $\mathrm{CD} 16^{+}$cells were $\mathrm{CD} 56^{-}$macrophages, and a low NK cell infiltration [37].

2.2. Tumor-Associated NK Cell Phenotype. The limited number of NK cells infiltrating tumors has hampered more extensive ex vivo analyses of such tumor-associated cells. However, novel studies of tumor-associated NK cells demonstrated a striking phenotype, supporting the notion that tumorinduced alterations of activating NK cell receptor expression may hamper immune surveillance and promote tumor progression. In this sense, several authors studied the balance between inhibitory and activating receptors of NK cells that infiltrate tumors, observing in some cases downregulation of activating receptors and in others, overexpression of inhibitory ones. In a study which analyzed the receptor repertoire and functional integrity of NK cells in peritoneal effusions from patients with ovarian carcinoma, tumorassociated NK cells expressed reduced levels of the activating DNAM-1, 2B4, and CD16 receptors and were hyporesponsive to HLA class I-deficient K562 cells and to coactivation via DNAM-1 and $2 \mathrm{~B} 4$, relative to autologous peripheral blood NK cells. Moreover, tumor-associated NK cells were 


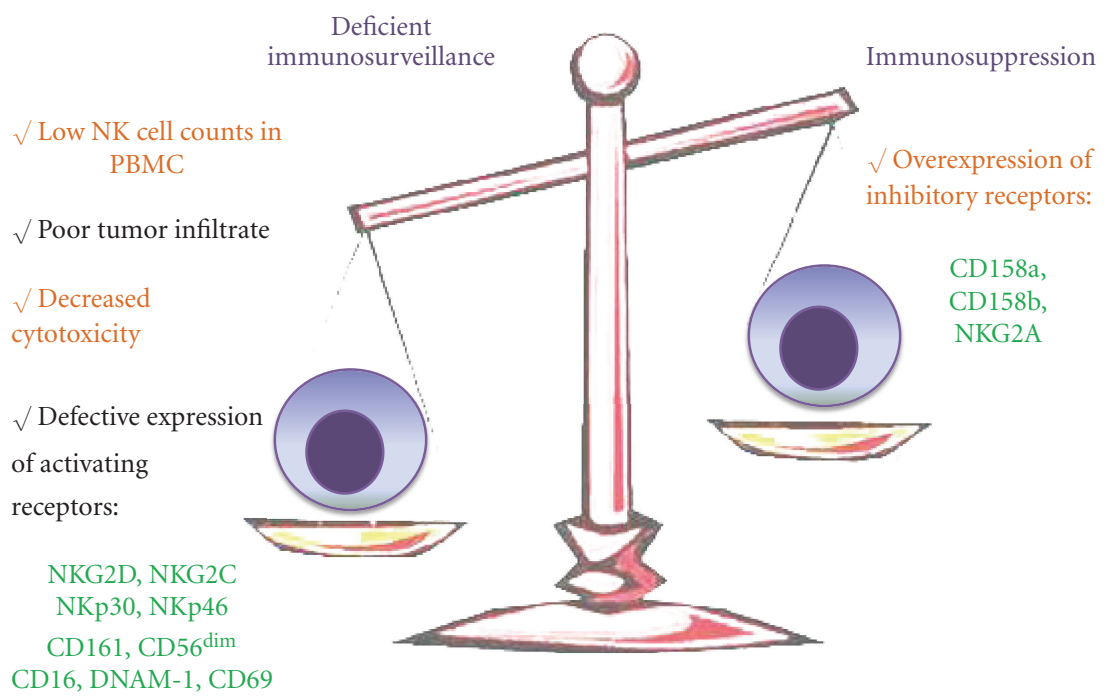

FIGURE 1: NK cell abnormalities in cancer patients. A defective immunity secondary to tumor development has been well established in different types of cancer. The imbalance of immune status is inclined to immunosuppression in cancer patients which results in a concomitant tendency to tumor immune evasion. Such immunosuppression is characterized by a decrease in NK cell numbers in PB and a decreased in tumor infiltrate as compared to normal tissue. Moreover, in many types of cancer an altered phenotype which presents defective expression of activating receptors and overexpression of inhibitory receptors is observed.

also refractory to CD16 receptor stimulation, resulting in diminished Ab-dependent cellular cytotoxicity (ADCC) against autologous tumor cells [38]. In another study, tongue cancer intraepithelial NK cells expressed NKG2A, an inhibitory receptor that recognizes HLA-E, more frequently than those in the stroma or in lichen planus. Collectively, the intraepithelial $\mathrm{CD} 8^{+} \mathrm{T}$ cells and NK cells were phenotypically inactivated, whereas stromal counterparts were phenotypically just as active as those in lichen planus, suggesting the suppressed state of the intraepithelial NK cells [39]. Carrega and coworkers described both relevant molecule expression and function of NK cells infiltrating NSCLC in comparison with autologous NK cells isolated from either peritumoral normal lung tissues or $\mathrm{PB}$. The $\mathrm{CD} 56^{\text {bright }} \mathrm{CD} 16^{-} \mathrm{NK}$ cell subset was highly enriched in tumor infiltrate and displayed activation markers, including NKp44, CD69, and HLA-DR. Remarkably, the cytolytic potential of NK cells isolated from cancer tissues was lower than that of NK cells from $\mathrm{PB}$ or normal lung tissue, whereas no difference was observed regarding their capability of producing cytokines [36]. In acute myeloid leukemia (AML) it was demonstrated that CD137 ligand (CD137L) was expressed on leukemic cells and interacts with CD137 on activated NK cells. Bidirectional signaling following CD137-CD137L interaction induced the release of the immunomodulatory cytokines IL-10 and TNF by AML cells and directly diminished granule mobilization, cytotoxicity, and IFN- $\gamma$ production by human NK cells, demonstrating an immune evasion of AML cells by impairing NK-cell tumor surveillance [40].

2.3. Peripheral Blood NK Phenotype. Not only did NK infiltrating tumor cells show a different phenotype or reduced cytotoxicity, but in some kind of cancers this was also observed in NK cells from PB. In a study in metastatic melanoma (MM) patients, NK cells presented decreased activity and IFN- $\gamma$ production and also had a redistribution of NK cell subsets. It was observed an increase in noncytotoxic $\mathrm{CD} 16^{\mathrm{dim}} \mathrm{CD} 56^{\text {bright }}$ and a reduction in cytotoxic CD16 ${ }^{\text {bright }}$ CD56 $6^{\text {dim }}$ NK cell subsets. Moreover, there was a decreased CD161 and NKG2D activating receptors and an overexpressed CD158a inhibitory NK cell receptor, which correlated to lower NK cell cytotoxicity [41]. These results are in agreement with another study in MM where a significantly lower percentage of NK cells expressed CD16, NKp30, and NKp46 activating receptors as compared to healthy donors. In addition, despite the importance of NKG2D in recognizing MM cells, no substantial differences were observed between stage IV MM patients and healthy donors [41]. These observations imply that down-regulated NK litic receptor expression in MM patients may affect NK cells ability to recognize and eliminate tumor cells [42]. Likewise, the frequency of NK cells expressing the activating receptors NKp30, NKp44, NKp46, NKG2D, and NKG2C was significantly decreased in AML patients compared to the NK cells of normal controls [43]. Although the molecular mechanisms responsible for the reduced receptor expression in PB NK cells remains elusive, elevated serum levels of soluble NK cell receptor ligands shed by tumor cells have been associated with downregulation of the NK cell receptors and might contribute to the decreased levels of NK cell activity [44].

In this section, we cited works which showed some impaired NK cells characteristics in cancer patients which determine their inability to eliminate tumor cells (depicted in Figure 1). On the other hand, it is a fact that NK 
cells are potentially active against tumor cells and it is feasible to manipulate them to restore and/or improve their antitumoral activity. NK cell-based immunotherapeutic approaches will be reviewed in next section.

\section{NK Cell Therapy}

3.1. Monoclonal Antibodies. Many mechanisms have been proposed to explain the clinical antitumor activity of unconjugated tumor antigen-specific monoclonal Abs (MAbs). The ability of some MAbs to disrupt signalling pathways involved in the maintenance of the malignant phenotype has received widespread attention. In addition, Abs exhibit various immunomodulatory properties and, by directly activating or inhibiting molecules of the immune system, they can promote the induction of antitumor immune responses. However, this ability has been less studied. In this section, we will describe NK cell-mediated ADCC mechanism and discuss the potential use of MAbs to manipulate the host immune response to tumors.

3.2. Antibody-Dependent Cellular Cytotoxicity. Abs are linked to immune effector functions by the Fc fragment, which is capable of initiating ADCC when binding to Fc receptors, specially Fc $\gamma$ RIII (CD16) on NK cells, which initiates a sequence of cellular events culminating in the release of cytotoxic granzyme-containing granules and INF- $\gamma$ secretion [45-47]. Several studies have established the importance of Fc-Fc $\gamma \mathrm{R}$ interactions for the antitumor effects of certain MAbs in murine models and clinical trials. A seminal paper showed that the antitumor activities of the anti-HER-2 MAb, Trastuzumab, and the anti-CD20 MAb, Rituximab, were lower in Fc $\gamma$ R-deficient mice than in wild-type mice [48]. The role of $\mathrm{F}_{\mathrm{c}} \gamma \mathrm{R}$ in the antitumor response has been further supported by the finding that polymorphisms in the gene encoding Fc $\gamma$ RIII, which lead to higher binding of $\mathrm{Ab}$ to Fc $\gamma$ RIII, are associated with high response rates to Rituximab in patients with follicular non-Hodgkin's lymphoma [27]. A separate study that compared clinical responses to Rituximab in patients with follicular lymphoma suggested that both Fc $\gamma$ RIII and Fc $\gamma$ RIIB have a role in the response to Rituximab [28]. More recent findings show that polymorphisms in genes encoding Fc $\gamma$ Rs are associated with clinical responses to other Abs, including Trastuzumab [29] and the antiEGFR MAb, Cetuximab [30]. Patients with breast cancer who responded to Trastuzumab with complete or partial remission have been found to have a higher capability to mediate in vitro ADCC in response to Trastuzumab than patients whose tumors failed to respond to therapy [29]. ADCC enhancement through Fc domain modification has shown promise in the development of next generation MAbs. For example, a CD19-specific MAb with increased Fc $\gamma$ RIIIA binding affinity mediated significantly increased ADCC compared to its parental MAb and Rituximab [49]. The in vivo infusion of this high affinity MAb efficiently cleared malignant B cells in cynomolgus macaques (Macaca fascicularis) [50].
3.3. Potentiating ADCC. In previous reports, we and others proved that NK cells produce Cetuximab-mediated ADCC of metastatic CRC (mCRC) and that this activity is not affected by the mutational status of the downstream molecule K-RAS $[51,52]$. This effect is expected because NK cells recognize the surface-bound Abs and are able to kill tumor cells independently of the EGFR pathway activation. Nevertheless, it remains to be answered why in mCRC patients with K-RAS mutated status Cetuximab-mediated ADCC does not induce clinical remissions. As we discussed, one of the possibilities is the low proportion of NK cells infiltrating CRC tumors [53] and the low functional capacity of these cells observed in cancer patients $[54,55]$. Therefore, if it were possible to enhance the activity of NK cells, the efficacy of treatment with Cetuximab could be increased. The anti-VEGF-A $\mathrm{MAb}$, Bevacizumab, is also active in mCRC, regardless of the K-RAS mutation status; nevertheless, it needs to be administered in combination with a cytotoxic agent [56]. Regarding ADCC enhancer molecules, Lenalidomide, an analog of thalidomide, is able to potentiate ADCC in vitro. Lenalidomide enhanced NK cell and monocyte-mediated ADCC of Rituximab against a variety of hematological cell lines in vitro [57]. Lenalidomide also enhanced NK cell-mediated lysis of Cetuximab and Trastuzumab-coated CRC and breast cancer cells, respectively. The ability of lenalidomide to enhance Cetuximab-mediated ADCC of CRC cells was not affected by the K-RAS mutational status [58]. Because panitumumab, an IgG2a anti-EGFR MAb, does not effectively interact with Fc $\gamma$ receptors on the NK cell surface, it was unable to initiate ADCC and, as expected, lenalidomide had no effect because its activity is reliant on the augmentation of NK cell signaling downstream of Fc $\gamma$ R. An early clinical study exploring this effect of lenalidomide in K-RAS mutant tumors has been initiated [59].

As explained previously, many studies demonstrated the impairment of NK cell activity in cancer patients. In these cases, cytokines could restore and potentiate NK cellmediated ADCC. The works cited below explain how these treatments work when patients present NK cell dysfunction. Cetuximab was tested in various in vitro studies in patients with esophageal squamous cell carcinoma (ESCC) to evaluate the possibility of treatment. Authors first performed detailed analysis of ADCC mediated by Cetuximab against ESCC cell lines with various levels of EGFR. The activities of Cetuximab-mediated ADCC by patients' PBMC were impaired in comparison with those by healthy donors' PBMC. Moreover, the inhibition of transforming growth factor (TGF) $\beta$ could enhance Cetuximab-mediated ADCC against TGF- $\beta$-producing ESCC [60]. In other work, downregulated CD16 and upregulated CD56 molecules on $\mathrm{NK}$ cells were observed in ESCC patients, resulting in NK cell dysfunction. After patients received curative resections of ESCC, the downregulated CD16 and upregulated CD56 were significantly restored to the levels of healthy donors. TGF$\beta 1$ was found to partially contribute to downregulation of CD16 on NK cells [61]. Watanabe and coworkers evaluated whether IL-21 could improve the impairment of ADCC in patients with ESCC as IL-21 was reported to have the 
ability to activate NK cells. Trastuzumab- and Cetuximabmediated ADCC of PBMC or of enriched NK cells was enhanced by the addition of IL-21 in a dose-dependent manner and the levels of ADCC enhanced by IL-21 in patients were high enough in comparison with those in healthy donors, paralleling the upregulation of CD247 on NK cells [55]. As in the previous example, NK cell response to Cetuximab-coated tumor cells could be enhanced by the administration of NK cell stimulatory cytokines IL-2, IL12 , or IL-21 and resulted in higher IFN- $\gamma$ production than was observed with either agent alone. NK cell-derived IFN- $\gamma$ significantly enhanced monocyte ADCC against Cetuximabcoated tumor cells. Costimulated NK cells also secreted elevated levels of chemokines (IL-8, macrophage inflammatory protein-1a, and RANTES) that could direct the migration of naive and activated T cells. Furthermore, administration of IL-21 enhanced the effects of Cetuximab in a murine tumor model [62]. In other experimental study, it was analyzed the correlation between EGFR expression in lung cancer cell lines and the ADCC activity of Cetuximab as well as the influence of IL-2 and chemotherapy on ADCC activity. A logarithmic correlation was observed between the number of EGFRs and ADCC activity. In addition, NK cell-mediated ADCC was enhanced by IL-2 and such cells were also less susceptible to immunosuppression by chemotherapy than in lung cancer patients [63]. The antitumor effect and mechanism of action of Cetuximab using EGFR high-expressing and EGFR lowexpressing gastric cancer cell lines without gene amplification was investigated. Cetuximab showed neither significant growth inhibition nor induction of apoptosis in either cell line in vitro, and only slightly inhibited ligand-induced phosphorylation of protein kinase B and extracellular signalregulated kinase. In contrast, Cetuximab significantly inhibited subcutaneous and intraperitoneal tumor growth in nude mice. This antitumor activity was significantly enhanced and diminished by treatment with IL-2 and antiasialo GM1 $\mathrm{Ab}$, respectively [64]. In that study, HER-2/neu-expressing gastric cancer cells could be killed by Herceptin, the antiHER-2/neu MAb. Herceptin-mediated ADCC correlated with the degree of HER-2/neu expression on the gastric cancer cells. However, the Herceptin-mediated ADCC was significantly impaired in PBMC from advanced disease patients compared with that in early disease or healthy individuals. Moreover, NK cells purified from patients with advanced disease indicated less Herceptin-mediated ADCC in comparison with that from healthy donors, whereas monocytes purified from the patients showed an almost equal amount of Herceptin-mediated ADCC in comparison with that from healthy individuals, indicating that NK cell dysfunction contributed to the impaired Herceptinmediated ADCC in gastric cancer patients. Furthermore, the NK-cell dysfunction on Herceptin-mediated ADCC correlated with the downregulation of CD16 expression in the patients, and IL-2 ex vivo treatment of NK cells could restore the impairment of Herceptin-mediated ADCC, concomitant to the normalization of the expression of CD16 molecules [65]. In our laboratory, we performed in vitro studies on human triple negative breast cancer (TNBC) K-RASmutated cell lines. We found that EGFR-expressing TNBC could be killed by Cetuximab-mediated ADCC at clinically achievable concentrations. Furthermore, IL-15 could replace IL-2 in most of its immunologic activities, stimulating NK cells ability to produce IFN- $\gamma$, paralleling the upregulation of activating receptors [66]. These results show that Cetuximabmediated NK cell activity can be significantly enhanced in the presence of NK cell stimulatory cytokines.

3.4. Adoptive Cell Transfer. Adoptive cell transfer (ACT) therapy can be considered as a strategy aimed at replacing, repairing, or enhancing the biological function of the immune system by means of autologous or allogeneic cells. ACT therapy may include (i) removal or enrichment of various cell populations; (ii) expansion of hematopoietic cell subsets; (iii) activation of lymphocytes for immunotherapy; (iv) genetic modification of lymphoid cells, when these cells are intended to engraft transiently in the recipient and/or be used in the treatment of cancer. This section contains extensive considerations on clinical and laboratory experience in immunologic targeting of malignant cell populations, and how lasting curative responses can be effectively generated. As we will discuss, the use of lymphocytes and/or NK cells as a strategic weapon in preventing or curing the neoplastic relapse after surgery/chemotherapy has been applied to the treatment of hematological malignancies and solid tumors.

NK cells represent a promising cell type to utilize for effective adoptive immunotherapy. Transfer of tumor-infiltrating lymphocytes has shown some remarkable responses in patients with advanced MM [67]. Nevertheless, problems with deriving sufficient numbers of these cells from patients, downregulation of $\mathrm{MHC}$ class I ligands and costimulatory ligands on tumor cells, combined with the lack of persistence of transferred cells have precluded broad utilization of these cells for the treatment of cancer patients. The transfer of other immune subsets, such as NK cells which do not require prior sensitization to respond to target cells and can potently induce a cytolytic response, represents a good alternate or auxiliary cell type for immunotherapy.

3.5. Ex Vivo Expansion. One of the main hurdles involved in developing adoptive transfer of immune cells has been to define good manufacturing procedures (GMPs) compliant methods to isolate defined subsets with the number of cells required that guarantee the safety of the injection to patients. In this respect, clinical-grade production of $\mathrm{NK}$ cells has proven efficient [68], and large-scale expansion method has been possible for human NK cells [69], using a cytokinebased culture system for ex vivo expansion of NK cells from hematopoietic progenitor cells from umbilical cord blood. Systematic refinement of this two-step system using a novel clinical-grade medium resulted in a therapeutically applicable cell culture protocol. Nevertheless, improved technologies for NK cells expansion lately have been tested. NK cells from myeloma patients expanded in a bioreactor displayed significantly higher cytotoxic capacity. It was possible to partially attribute this to a higher expression level of NKp44 compared with NK cells expanded in flasks [70]. These results demonstrate that large amounts of highly active 
NK cells for adoptive immunotherapy can be produced in a closed, automated, large-scale bioreactor under feeder-free current GMP conditions, facilitating clinical trials for the use of these cells.

Even when the first works were based on transplantation of autologous ex vivo expanded cells, and in addition to what we earlier discussed on cancer patients NK cells dysfunctions, it is now understood that the failure of autologous NK therapy is partially due to the downregulation of $\mathrm{NK}$ cell killing that occurs with recognition of self-class I MHC on tumor cells [71-73], making allogeneic cell transfer more attractive. During allogeneic hematopoietic stem cell transplantation, NK cells have been implicated in the suppression of Graft versus Host Disease (GVHD), the promotion of bone marrow engraftment, and mediation of a Graft versus Leukemia (GVL) effect [74]. In the setting of allogeneic ACT in leukemias, the results of NK cells activity depend on the directionality of lysis $[75,76]$. When the NK cells are donorderived and the recipient cells lack expression of the cognate KIR ligand, the donor NK cell lysis of recipient target cells can result in GVL and/or GVHD, depending on the tissue origin of the NK cell target. However, if the target cell is of donor origin, and the NK effector cell is of recipient origin, NK cell lysis can result in graft rejection. Recent studies have demonstrated that NK cell effector capacity is influenced by class and quantity of inhibitory receptors for self-HLA$\mathrm{B}$ and HLA-C ligands [77, 78]. It has been estimated that NK cell alloreactivity can be expected to occur in about $50 \%$ of unrelated donor transplants with one or more HLA allele level mismatches. Velardi and coworkers announced a new era in the exploitation of NK cells for cancer immunotherapy with a pioneering study of hematopoietic stem cell transplantation that stratified patients according to KIR-ligand mismatch between donor cells and recipient leukemic cells [74]. This reveals that alloreactive NK cells, unrestrained by inhibitory signals from the recipient HLA ligands, protects against disease relapse. This KIR-ligand mismatch phenomenon has attracted researchers to study it, and similar observations have since been made in other trials of leukemia immunotherapy [79-81]. On the other hand, further studies extended HLA match analysis to the mechanisms involved in GVHD. In patients with hematologic malignancies who received transplants from unrelated donors, genotype analysis of six major HLA loci identified 4 HLA-C and 6 HLADPB1 mismatch combinations responsible for a decreased risk of relapse and severe acute GVHD. Donor selection made in consideration of these results might allow the separation of GVL from acute GVHD, especially in HLA-DPB1 mismatch combinations [82]. These findings might be crucial to elucidating the mechanism of the decreased risk of relapse on the basis of HLA molecule haplotype (Figure 2).

The effectiveness of adoptive transfer of expanded NK cells for the treatment of relapsed leukemia has been demonstrated, and ongoing efforts are designed to evaluate this approach in the treatment of solid tumor malignancies as well [83-85]. Despite this therapy has been proven safe for patients and feasible, more effective strategies to augment in vivo NK cell persistence and expansion are needed to test the clinical benefit of NK cells against solid tumors, as literature supports the notion that there is a cell dose response in ACT. In a phase II clinical trial in patients with ovarian and breast cancer, the adoptive transfer of haploidentical NK cells after lymphodepleting chemotherapy was associated with transient donor chimerism that was not improved with the addition of low-dose total body irradiation. Sustained in vivo NK cell expansion may be limited by host rejection, competition with host lymphocytes or suppression by recipient regulatory $\mathrm{T}$ cells (Treg) or myeloderived suppressor cells [86]. To provide a greater number of NK cells with a differentiated activated phenotype for adoptive immunotherapy, some authors described cytokinebased methods useful for clinical-scale NK cell production. A phase I clinical study evaluated the safety of donor lymphocyte infusions following allogeneic hematopoietic stem cell transplantation to patients with progressive malignant disease. This study reported the safety of ex vivo-expanded NK and NK-like T cells with a feeder-free cGMP compatible expansion strategy administered to humans also in combination with IL-2 [87]. Decot and coworkers described an immunomagnetic technique consisting in CD3/CD19 depletion effective to obtain highly $\mathrm{T}$ - and B-cell-depleted NK cell-enriched product with GMP-compliant reagents. IL-2 or IL-15 were equivalent to significantly enhance NK cell cytotoxicity after culture with MHC-class I negative erythroleukemia cell line. Furthermore, they observed a modification in NK-cell receptor expression pattern with upregulation of the activating receptor NKG2D, but also of the inhibiting receptors KIR2DL1 and KIR2DL2 [88]. In other work PBMC were cultured in serum-free medium and IL-2 for 20 days. Cells in the culture were also stimulated with anti-CD3 Ab (OKT3). Safety and feasibility of administering ex vivo expanded NK and NK-like T-effector cells as donor lymphocytes infusion to five cancer patients after stem cell transplantation was evaluated. Cell infusions, with or without IL-2 injections, seemed to be safe as no GVHD was observed. Not only do all these data support a role of NK cell during ACT therapy, but results also link NK cell recovery with a GVL rather than with a GVHD effect, as recipient's NK cells have been shown to be the first of lymphoid lineage cells to reconstitute following allogeneic transplantation, and adequate recovery of NK cell number in the early posttransplant period has been associated with improved relapse-free outcome [89].

These studies now provide the backdrop for the development of therapies that enhance the therapeutic benefit of allogeneic transplantation while minimizing the risks and toxicities associated with treatment (Figure 3).

3.6. Concluding Remarks. In recent years there have been significant advances in the discovery of the molecular mechanisms that govern the reactivity of NK cells against tumors. In this paper we have reviewed how the expression and function of both inhibitory and activating NK receptors are involved in the molecular regulation of innate immunity in malignant settings.

NK cells are also potential tools for cancer therapy, both due to cytotoxic ability enhanced by antibodies and 


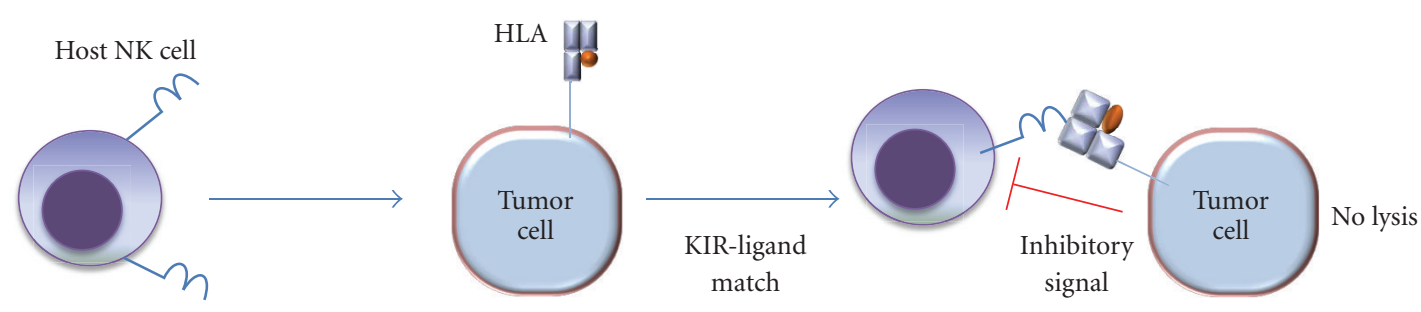

KIR

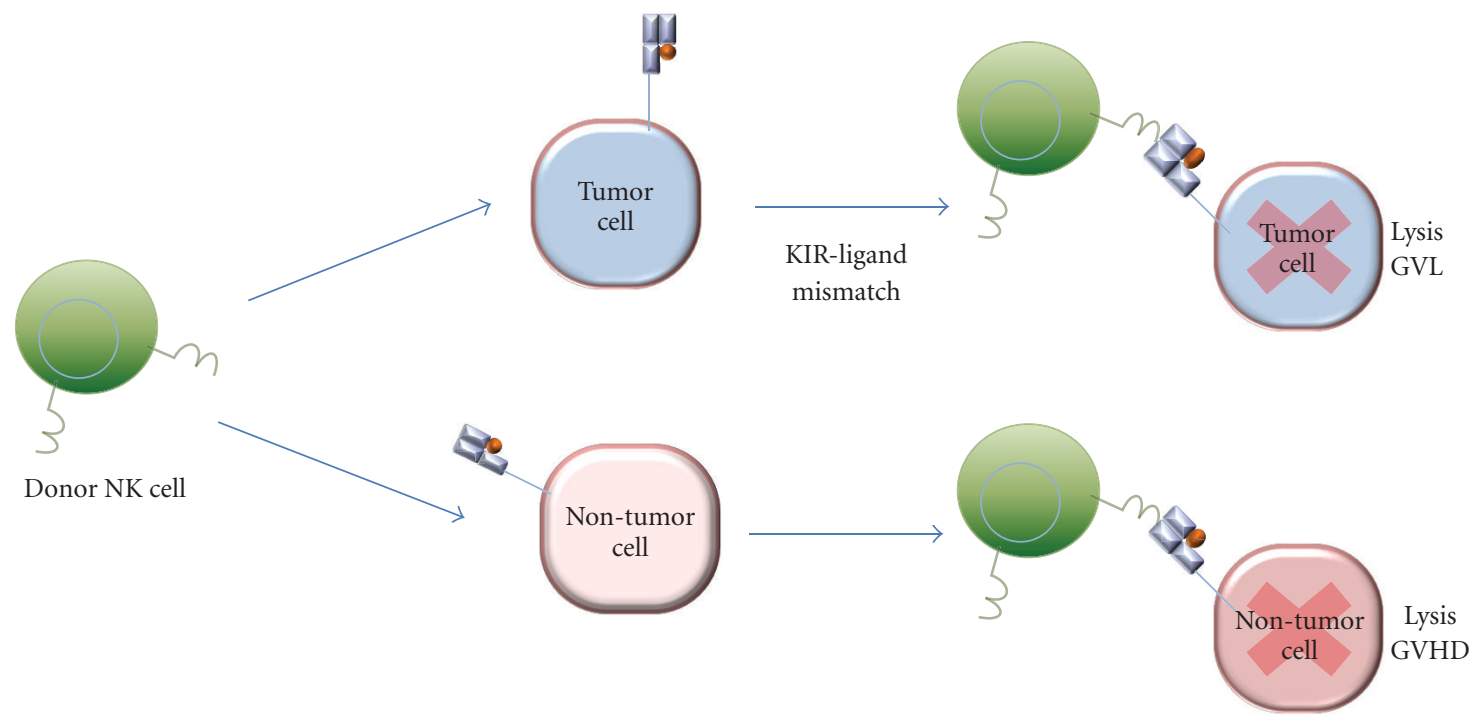

FigURE 2: NK cell tumor immunity and alloimmunity after allogeneic ACT. Autologous NK cell killing is inhibited when self-class I MHC on tumor cells is recognized. In allogeneic ACT, NK cell KIR-mismatch is implicated both in GVHD and in mediating of a GVL effect.

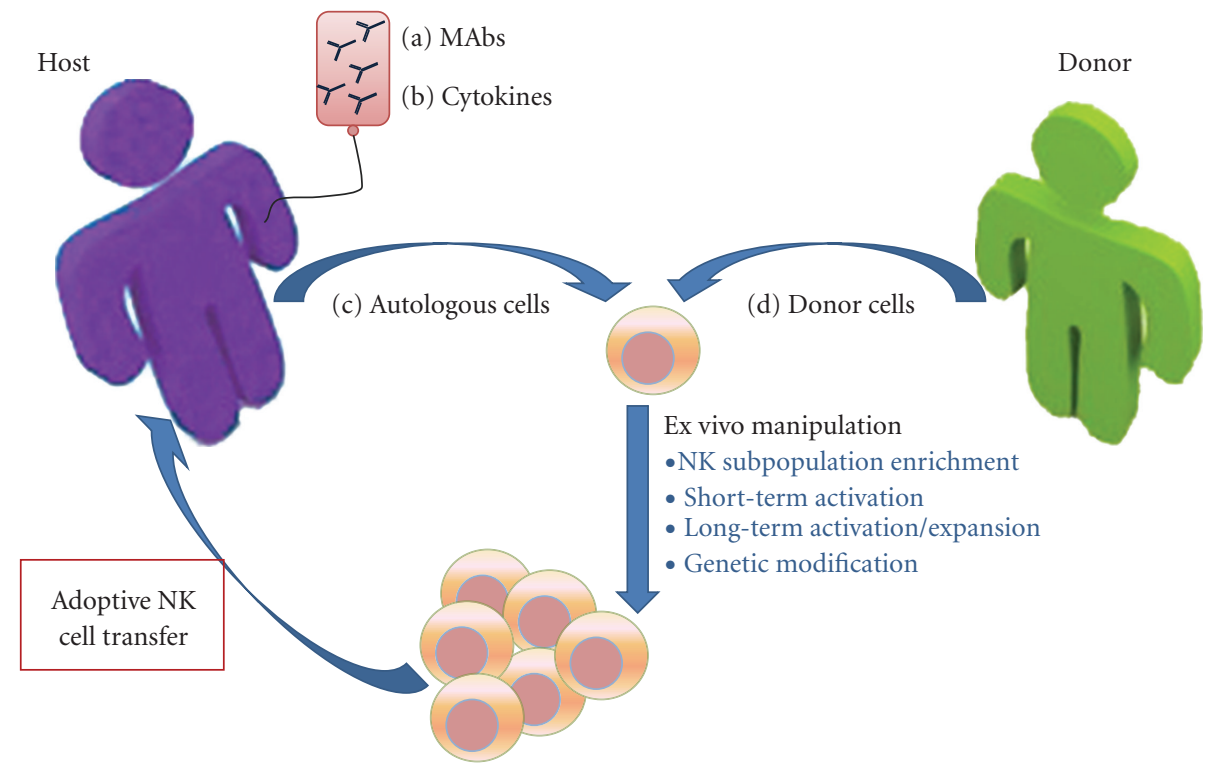

FigURE 3: NK cell-based immunotherapies. Overview of current approaches being tested in clinical settings. (a) Infusion of tumor-specific MAbs triggers an ADCC response. (b) ADCC can be potentiated with the coadministration of cytokines. (c) Autologous and (d) allogeneic transfer of NK cells, which can be manipulated ex vivo with different approaches. 
the possibility of ex vivo expansion and adoptive transfer. Nevertheless therapeutical regimens need to be further developed. Inasmuch as one scheme will not be suitable for all malignancies, different approaches are to be evaluated for each particular case.

\section{Conflicts of Interests}

authors declared that there are no conflict of interests.

\section{Acknowledgments}

This work was supported with funds from Fundación Sales, Fundación P. Mosoteguy, Fundación Cáncer (FUCA), Agencia Nacional de Promoción Científica y Tecnológica (ANPCyT), and Fundación María Calderón de la Barca. J. Mordoh, and E. M. Levy are members of CONICET.

\section{References}

[1] E. Vivier, E. Tomasello, M. Baratin, T. Walzer, and S. Ugolini, "Functions of natural killer cells," Nature Immunology, vol. 9, no. 5, pp. 503-510, 2008.

[2] G. Trinchieri, "Biology of natural killer cells," Advances in Immunology, vol. 47, pp. 187-376, 1989.

[3] S. Kim, K. Iizuka, H. L. Aguila, I. L. Weissman, and W. M. Yokoyama, "In vivo natural killer cell activities revealed by natural killer cell-deficient mice," Proceedings of the National Academy of Sciences of the United States of America, vol. 97, no. 6, pp. 2731-2736, 2000.

[4] M. J. Smyth, Y. Hayakawa, K. Takeda, and H. Yagita, "New aspects of natural-killer-cell surveillance and therapy of cancer," Nature Reviews Cancer, vol. 2, no. 11, pp. 850-861, 2002.

[5] J. Wu and L. L. Lanier, "Natural killer cells and cancer," Advances in Cancer Research, vol. 90, pp. 127-156, 2003.

[6] C. A. Stewart and E. Vivier, "Strategies of NK cell recognition and their roles in tumor immunosurveillance," in How the Immune System Recognizes Self and Nonself: Immunoreceptors and Their Signaling, D. Kitamura, Ed., pp. 37-81, Springer, Tokyo, Japan, 2007.

[7] D. H. Kaplan, V. Shankaran, A. S. Dighe et al., "Demonstration of an interferon $\gamma$-dependent tumor surveillance system in immunocompetent mice," Proceedings of the National Academy of Sciences of the United States of America, vol. 95, no. 13, pp. 7556-7561, 1998.

[8] V. Shankaran, H. Ikeda, A. T. Bruce et al., "IFN $\gamma$, and lymphocytes prevent primary tumour development and shape tumour immunogenicity," Nature, vol. 410, no. 6832, pp. 1107-1111, 2001.

[9] C. M. Koebel, W. Vermi, J. B. Swann et al., "Adaptive immunity maintains occult cancer in an equilibrium state," Nature, vol. 450, no. 7171, pp. 903-907, 2007.

[10] K. Karre, H. G. Ljunggren, G. Piontek, and R. Kiessling, "Selective rejection of $\mathrm{H}$-2-deficient lymphoma variants suggests alternative immune defence strategy," Nature, vol. 319, no. 6055, pp. 675-678, 1986.

[11] A. Diefenbach, E. R. Jensen, A. M. Jamieson, and D. H. Raulet, "Rae1 and H60 ligands of the NKG2D receptor stimulate tumour immunity," Nature, vol. 413, no. 6852, pp. 165-171, 2001.
[12] A. Cerwenka, J. L. Baron, and L. L. Lanier, "Ectopic expression of retinoic acid early inducible-1 gene (RAE-1) permits natural killer cell-mediated rejection of a MHC class I-bearing tumor in vivo," Proceedings of the National Academy of Sciences of the United States of America, vol. 98, no. 20, pp. 1152111526, 2001.

[13] J. M. Kelly, P. K. Darcy, J. L. Markby et al., "Induction of tumor-specific T cell memory by NK cell-mediated tumor rejection," Nature Immunology, vol. 3, no. 1, pp. 83-90, 2002.

[14] J. C. Sun and L. L. Lanier, "Natural killer cells remember: an evolutionary bridge between innate and adaptive immunity?" European Journal of Immunology, vol. 39, no. 8, pp. 2059-2064, 2009.

[15] S. E. A. Street, Y. Hayakawa, Y. Zhan et al., "Innate Immune Surveillance of Spontaneous B Cell Lymphomas by Natural Killer Cells and $\gamma \delta$ T Cells," Journal of Experimental Medicine, vol. 199, no. 6, pp. 879-884, 2004.

[16] C. Y. Koh, B. R. Blazar, T. George et al., "Augmentation of antitumor effects by NK cell inhibitory receptor blockade in vitro and in vivo," Blood, vol. 97, no. 10, pp. 3132-3137, 2001.

[17] J. S. Orange, "Human natural killer cell deficiencies," Current Opinion in Allergy and Clinical Immunology, vol. 6, no. 6, pp. 399-409, 2006.

[18] K. Imai, S. Matsuyama, S. Miyake, K. Suga, and K. Nakachi, "Natural cytotoxic activity of peripheral-blood lymphocytes and cancer incidence: an 11-year follow-up study of a general population," The Lancet, vol. 356, no. 9244, pp. 1795-1799, 2000.

[19] S. Coca, J. Perez-Piqueras, D. Martinez et al., "The prognostic significance of intratumoral natural killer cells in patients with colerectal carcinoma," Cancer, vol. 79, no. 12, pp. 2320-2328, 1997.

[20] S. Ishigami, S. Natsugoe, K. Tokuda et al., "Prognostic value of intratumoral natural killer cells in gastric carcinoma," Cancer, vol. 88, no. 3, pp. 577-583, 2000.

[21] F. R. Villegas, S. Coca, V. G. Villarrubia et al., "Prognostic significance of tumor infiltrating natural killer cells subset CD57 in patients with squamous cell lung cancer," Lung Cancer, vol. 35, no. 1, pp. 23-28, 2002.

[22] P. A. Albertsson, P. H. Basse, M. Hokland et al., "NK cells and the tumour microenvironment: implications for NK-cell function and anti-tumour activity," Trends in Immunology, vol. 24, no. 11, pp. 603-609, 2003.

[23] G. Esendagli, K. Bruderek, T. Goldmann et al., "Malignant and non-malignant lung tissue areas are differentially populated by natural killer cells and regulatory $\mathrm{T}$ cells in non-small cell lung cancer," Lung Cancer, vol. 59, no. 1, pp. 32-40, 2008.

[24] M. Hokland, J. Kjaergaard, P. J. K. Kuppen et al., "Endogenous and adoptively transferred A-NK and T-LAK cells continuously accumulate within murine metastases up to $48 \mathrm{~h}$ after inoculation," In Vivo, vol. 13, no. 3, pp. 199-204, 1999.

[25] K. C. Hsu, C. A. Keever-Taylor, A. Wilton et al., "Improved outcome in HLA-identical sibling hematopoietic stem-cell transplantation for acute myelogenous leukemia predicted by KIR and HLA genotypes," Blood, vol. 105, no. 12, pp. 48784884, 2005.

[26] L. Ruggeri, A. Mancusi, E. Burchielli, F. Aversa, M. F. Martelli, and A. Velardi, "Natural killer cell alloreactivity in allogeneic hematopoietic transplantation," Current Opinion in Oncology, vol. 19, no. 2, pp. 142-147, 2007.

[27] G. Cartron, L. Dacheux, G. Salles et al., "Therapeutic activity of humanized anti-CD20 monoclonal antibody and polymorphism in IgG Fc receptor Fc $y$ rIIIa gene," Blood, vol. 99, no. 3, pp. 754-758, 2002. 
[28] W. K. Weng and R. Levy, "Two immunoglobulin G fragment $\mathrm{C}$ receptor polymorphisms independently predict response to rituximab in patients with follicular lymphoma," Journal of Clinical Oncology, vol. 21, no. 21, pp. 3940-3947, 2003.

[29] A. Musolino, N. Naldi, B. Bortesi et al., "Immunoglobulin $\mathrm{g}$ fragment $\mathrm{c}$ receptor polymorphisms and clinical efficacy of trastuzumab-based therapy in patients with HER-2/neupositive metastatic breast cancer," Journal of Clinical Oncology, vol. 26, no. 11, pp. 1789-1796, 2008.

[30] F. Bibeau, E. Lopez-Crapez, F. D. Fiore et al., "Impact of fc $\gamma$ RIIa-fc $\gamma$ RIIIa polymorphisms and KRAS mutations on the clinical outcome of patients with metastatic colorectal cancer treated with cetuximab plus irinotecan," Journal of Clinical Oncology, vol. 27, no. 7, pp. 1122-1129, 2009.

[31] L. Zitvogel, A. Tesniere, and G. Kroemer, "Cancer despite immunosurveillance: immunoselection and immunosubversion," Nature Reviews Immunology, vol. 6, no. 10, pp. 715-727, 2006.

[32] Y. Naito, K. Saito, K. Shiiba et al., "CD8+ T cells infiltrated within cancer cell nests as a prognostic factor in human colorectal cancer," Cancer Research, vol. 58, no. 16, pp. 34913494, 1998.

[33] E. Sato, S. H. Olson, J. Ahn et al., "Intraepithelial CD8+ tumor-infiltrating lymphocytes and a high CD8/regulatory $\mathrm{T}$ cell ratio are associated with favorable prognosis in ovarian cancer," Proceedings of the National Academy of Sciences of the United States of America, vol. 102, no. 51, pp. 18538-18543, 2005.

[34] J. Galon, A. Costes, F. Sanchez-Cabo et al., "Type, density, and location of immune cells within human colorectal tumors predict clinical outcome," Science, vol. 313, no. 5795, pp. 19601964, 2006.

[35] M. Gulubova, I. Manolova, D. Kyurkchiev, A. Julianov, and I. Altunkova, "Decrease in intrahepatic CD56 ${ }^{+}$lymphocytes in gastric and colorectal cancer patients with liver metastases," Acta Pathologica, Microbiologica et Immunologica, vol. 117, no. 12, pp. 870-879, 2009.

[36] P. Carrega, B. Morandi, R. Costa et al., "Natural killer cells infiltrating human nonsmall-cell lung cancer are enriched in CD56 bright CD16- cells and display an impaired capability to kill tumor cells," Cancer, vol. 112, no. 4, pp. 863-875, 2008.

[37] G. Sconocchia, G. C. Spagnoli, D. Del Principe et al., "Defective infiltration of natural killer cells in MICA/Bpositive renal cell carcinoma involves $\beta$-integrin-mediated interaction,” Neoplasia, vol. 11, no. 7, pp. 662-671, 2009.

[38] M. Carlsten, H. Norell, Y. T. Bryceson et al., "Primary human tumor cells expressing CD155 impair tumor targeting by down-regulating DNAM-1 on NK cells," Journal of Immunology, vol. 183, no. 8, pp. 4921-4930, 2009.

[39] F. Katou, H. Ohtani, Y. Watanabe, T. Nakayama, O. Yoshie, and K. Hashimoto, "Differing phenotypes between intraepithelial and stromal lymphocytes in early-stage tongue cancer," Cancer Research, vol. 67, no. 23, pp. 11195-11201, 2007.

[40] T. Baessler, J. E. Charton, B. J. Schmiedel et al., "CD137 ligand mediates opposite effects in human and mouse NK cells and impairs NK-cell reactivity against human acute myeloid leukemia cells," Blood, vol. 115, no. 15, pp. 3058-3069, 2010.

[41] G. Konjević, K. Miräjaić Martinovi, V. Jurišić, N. Babović, and I. Spužić, "Biomarkers of suppressed natural killer (NK) cell function in metastatic melanoma: decreased NKG2D and increased CD158a receptors on CD3-CD16+ NK cells," Biomarkers, vol. 14, no. 4, pp. 258-270, 2009.

[42] G. Markel, R. Seidman, M. J. Besser et al., "Natural killer lysis receptor (NKLR)/NKLR-ligand matching as a novel approach for enhancing anti-tumor activity of allogeneic NK cells," PLoS ONE, vol. 4, no. 5, Article ID e5597, 2009.

[43] M. J. Szczepanski, M. Szajnik, A. Welsh, K. A. Foon, T. L. Whiteside, and M. Boyiadzis, "Interleukin-15 enhances natural killer cell cytotoxicity in patients with acute myeloid leukemia by upregulating the activating NK cell receptors," Cancer Immunology, Immunotherapy, vol. 59, no. 1, pp. 73-79, 2010.

[44] M. B. Fuertes, M. V. Girart, L. L. Molinero et al., "Intracellular retention of the NKG2D ligand MHC class i chain-related gene a in human melanomas confers immune privilege and prevents NK cell-mediated cytotoxicity," Journal of Immunology, vol. 180, no. 7, pp. 4606-4614, 2008.

[45] P. J. Leibson, "Signal transduction during natural killer cell activation: inside the mind of a killer," Immunity, vol. 6, no. 6, pp. 655-661, 1997.

[46] H. Terunuma, X. Deng, Z. Dewan, S. Fujimoto, and N. Yamamoto, "Potential role of NK cells in the induction of immune responses: implications for NK cell-based immunotherapy for cancers and viral infections," International Reviews of Immunology, vol. 27, no. 3, pp. 93-110, 2008.

[47] N. Anfossi, P. André, S. Guia et al., "Human NK cell education by inhibitory receptors for MHC class I," Immunity, vol. 25, no. 2, pp. 331-342, 2006.

[48] R. A. Clynes, T. L. Towers, L. G. Presta, and J. V. Ravetch, "Inhibitory $\mathrm{Fc}$ receptors modulate in vivo cytoxicity against tumor targets," Nature Medicine, vol. 6, no. 4, pp. 443-446, 2000.

[49] H. M. Horton, M. J. Bernett, E. Pong et al., "Potent in vitro and in vivo activity of an Fc-engineered anti-CD19 monoclonal antibody against lymphoma and leukemia," Cancer Research, vol. 68, no. 19, pp. 8049-8057, 2008.

[50] J. Zalevsky, I. W. L. Leung, S. Karki et al., "The impact of Fc engineering on an anti-CD19 antibody: increased Fc $\gamma$ receptor affinity enhances B-cell clearing in nonhuman primates," Blood, vol. 113, no. 16, pp. 3735-3743, 2009.

[51] E. M. Levy, G. Sycz, J. M. Arriaga et al., "Cetuximab-mediated cellular cytotoxicity is inhibited by HLA-E membrane expression in colon cancer cells," Innate Immunity, vol. 15, no. 2, pp. 91-100, 2009.

[52] P. Correale, M. Marra, C. Remondo et al., "Cytotoxic drugs up-regulate epidermal growth factor receptor (EGFR) expression in colon cancer cells and enhance their susceptibility to EGFR-targeted antibody-dependent cell-mediatedcytotoxicity (ADCC)," European Journal of Cancer, vol. 46, no. 9, pp. 1703-1711, 2010.

[53] F. Pagès, A. Kirilovsky, B. Mlecnik et al., "In situ cytotoxic and memory $\mathrm{T}$ cells predict outcome in patients with early-stage colorectal cancer," Journal of Clinical Oncology, vol. 27, no. 35, pp. 5944-5951, 2009.

[54] S. Wulff, R. Pries, K. Börngen, T. Trenkle, and B. Wollenberg, "Decreased levels of circulating regulatory NK cells in patients with head and neck cancer throughout all tumor stages," Anticancer Research, vol. 29, no. 8, pp. 3053-3057, 2009.

[55] M. Watanabe, K. Kono, Y. Kawaguchi et al., "Interleukin21 can efficiently restore impaired antibody-dependent cellmediated cytotoxicity in patients with oesophageal squamous cell carcinoma," British Journal of Cancer, vol. 102, no. 3, pp. 520-529, 2010.

[56] H. I. Hurwitz, J. Yi, W. Ince, W. F. Novotny, and O. Rosen, “The clinical benefit of bevacizumab in metastatic colorectal cancer is independent of K-ras mutation status: analysis of a phase in study of bevacizumab with chemotherapy in previously 
untreated metastatic colorectal cancer," Oncologist, vol. 14, no. 1, pp. 22-28, 2009.

[57] L. Wu, M. Adams, T. Carter et al., "Lenalidomide enhances natural killer cell and monocyte-mediated antibodydependent cellular cytotoxicity of rituximab-treated CD20+ tumor cells," Clinical Cancer Research, vol. 14, no. 14, pp. 4650-4657, 2008.

[58] L. Wu, A. Parton, L. Lu, M. Adams, P. Schafer, and J. B. Bartlett, "Lenalidomide enhances antibody-dependent cellular cytotoxicity of solid tumor cells in vitro: influence of host immune and tumor markers," Cancer Immunology, Immunotherapy, vol. 60, no. 1, pp. 61-73, 2011.

[59] D. Keizman, M. Zahurak, V. Sinibaldi et al., "Lenalidomide in nonmetastatic biochemically relapsed prostate cancer: results of a phase I/II double-blinded, randomized study," Clinical Cancer Research, vol. 16, no. 21, pp. 5269-5276, 2010.

[60] Y. Kawaguchi, K. Kono, K. Mimura, H. Sugai, H. Akaike, and H. Fujii, "Cetuximab induce antibody-dependent cellular cytotoxicity against EGFR-expressing esophageal squamous cell carcinoma," International Journal of Cancer, vol. 120, no. 4, pp. 781-787, 2007.

[61] M. Watanabe, K. Kono, Y. Kawaguchi et al., "NK cell dysfunction with down-regulated CD16 and up-regulated CD56 molecules in patients with esophageal squamous cell carcinoma," Diseases of the Esophagus, vol. 23, no. 8, pp. 675$681,2010$.

[62] J. M. Roda, T. Joshi, J. P. Butchar et al., "The activation of natural killer cell effector functions by cetuximab-coated, epidermal growth factor receptor-positive tumor cells is enhanced by cytokines," Clinical Cancer Research, vol. 13, no. 21, pp. 6419-6428, 2007.

[63] J. Kurai, H. Chikumi, K. Hashimoto et al., "Antibodydependent cellular cytotoxicity mediated by cetuximab against lung cancer cell lines," Clinical Cancer Research, vol. 13, no. 5, pp. 1552-1561, 2007.

[64] M. Hara, H. Nakanishi, K. Tsujimura et al., "Interleukin2 potentiation of cetuximab antitumor activity for epidermal growth factor receptor-overexpressing gastric cancer xenografts through antibody-dependent cellular cytotoxicity," Cancer Science, vol. 99, no. 7, pp. 1471-1478, 2008.

[65] K. Kono, A. Takahashi, F. Ichihara, H. Sugai, H. Fujii, and Y. Matsumoto, "Impaired antibody-dependent cellular cytotoxicity mediated by Herceptin in patients with gastric cancer," Cancer Research, vol. 62, no. 20, pp. 5813-5817, 2002.

[66] M. P. Roberti, M. M. Barrio, A. I. Bravo et al., "IL-15 and IL2 increase Cetuximab-mediated cellular cytotoxicity against triple negative breast cancer cell lines expressing EGFR," Breast Cancer Research and Treatment. In press.

[67] M. E. Dudley, J. R. Wunderlich, P. F. Robbins et al., "Cancer regression and autoimmunity in patients after clonal repopulation with antitumor lymphocytes," Science, vol. 298, no. 5594, pp. 850-854, 2002.

[68] D. H. McKenna, D. Sumstad, N. Bostrom et al., "Good manufacturing practices production of natural killer cells for immunotherapy: a six-year single-institution experience," Transfusion, vol. 47, no. 3, pp. 520-528, 2007.

[69] J. Spanholtz, M. Tordoir, D. Eissens et al., "High log-scale expansion of functional human natural killer cells from umbilical cord blood CD34-positive cells for adoptive cancer immunotherapy," PLoS ONE, vol. 5, no. 2, Article ID e9221, 2010.

[70] T. Sutlu, B. Stellan, M. Gilljam et al., "Clinical-grade, largescale, feeder-free expansion of highly active human natural killer cells for adoptive immunotherapy using an automated bioreactor," Cytotherapy, vol. 12, no. 8, pp. 1044-1055, 2010.

[71] D. H. Raulet and W. Held, "Natural killer cell receptors: the offs and ons of NK cell recognition," Cell, vol. 82, no. 5, pp. 697-700, 1995.

[72] K. Karre, "Express yourself or die: peptides, MHC molecules, and NK cells," Science, vol. 267, no. 5200, pp. 978-979, 1995.

[73] A. Moretta, M. Vitale, C. Bottino et al., "P58 molecules as putative receptors for major histocompatibility complex (MHC) class I molecules in human natural killer (NK) cells. Anti-p58 antibodies reconstitute lysis of MHC class Iprotected cells in NK clones displaying different specificities," Journal of Experimental Medicine, vol. 178, no. 2, pp. 597-604, 1993.

[74] L. Ruggeri, M. Capanni, E. Urbani et al., "Effectiveness of donor natural killer cell aloreactivity in mismatched hematopoietic transplants," Science, vol. 295, no. 5562, pp. 2097-2100, 2002.

[75] K. C. Hsu and B. O. Dupont, "Natural killer cell receptors: regulating innate immune responses to hematologic malignancy," Seminars in Hematology, vol. 42, no. 2, pp. 91-103, 2005.

[76] L. Ruggeri, M. Capanni, A. Mancusi, M. F. Martelli, and A. Velardi, "The impact of donor natural killer cell alloreactivity on allogeneic hematopoietic transplantation," Transplant Immunology, vol. 14, no. 3-4, pp. 203-206, 2005.

[77] M. Pfeiffer, M. Schumm, T. Feuchtinger, K. Dietz, R. Handgretinger, and P. Lang, "Intensity of HLA class I expression and KIR-mismatch determine NK-cell mediated lysis of leukaemic blasts from children with acute lymphatic leukaemia," British Journal of Haematology, vol. 138, no. 1, pp. 97-100, 2007.

[78] J. Yu, G. Heller, J. Chewning, S. Kim, W. M. Yokoyama, and K. C. Hsu, "Hierarchy of the human natural killer cell response is determined by class and quantity of inhibitory receptors for self-HLA-B and HLA-C ligands," Journal of Immunology, vol. 179, no. 9, pp. 5977-5989, 2007.

[79] S. Giebel, F. Locatelli, T. Lamparelli et al., "Survival advantage with KIR ligand incompatibility in hematopoietic stem cell transplantation from unrelated donors," Blood, vol. 102, no. 3, pp. 814-819, 2003.

[80] L. Ruggeri, A. Mancusi, K. Perruccio, E. Burchielli, M. F. Martelli, and A. Velardi, "Natural killer cell alloreactivity for leukemia therapy," Journal of Immunotherapy, vol. 28, no. 3, pp. 175-182, 2005.

[81] D. W. Beelen, H. D. Ottinger, S. Ferencik et al., "Genotypic inhibitory killer immunoglobulin-like receptor ligand incompatibility enhances the long-term antileukemic effect of unmodified allogeneic hematopoietic stem cell transplantation in patients with myeloid leukemias," Blood, vol. 105, no. 6, pp. 2594-2600, 2005.

[82] T. Kawase, K. Matsuo, K. Kashiwase et al., "HLA mismatch combinations associated with decreased risk of relapse: implications for the molecular mechanism," Blood, vol. 113, no. 12, pp. 2851-2858, 2009.

[83] D. Cho, D. R. Shook, N. Shimasaki, Y.-H. Chang, H. Fujisaki, and D. Campana, "Cytotoxicity of activated natural killer cells against pediatric solid tumors," Clinical Cancer Research, vol. 16, no. 15, pp. 3901-3909, 2010.

[84] S. Arai, R. Meagher, M. Swearingen et al., "Infusion of the allogeneic cell line NK-92 in patients with advanced renal cell cancer or melanoma: a phase I trial," Cytotherapy, vol. 10, no. 6, pp. 625-632, 2008.

[85] E. G. Iliopoulou, P. Kountourakis, M. V. Karamouzis et al., "A phase i trial of adoptive transfer of allogeneic natural killer cells in patients with advanced non-small cell lung cancer," 
Cancer Immunology, Immunotherapy, vol. 59, no. 12, pp. 17811789, 2010.

[86] M. A. Geller, S. Cooley, P. L. Judson et al., "A phase II study of allogeneic natural killer cell therapy to treat patients with recurrent ovarian and breast cancer," Cytotherapy, vol. 13, no. 1, pp. 98-107, 2010.

[87] L. Barkholt, E. Alici, R. Conrad et al., "Safety analysis of ex vivo-expanded NK and NK-like T cells administered to cancer patients: a Phase I clinical study," Immunotherapy, vol. 1, no. 5, pp. 753-764, 2009.

[88] V. Decot, L. Voillard, V. Latger-Cannard et al., "Natural-killer cell amplification for adoptive leukemia relapse immunotherapy: comparison of three cytokines, IL-2, IL-15, or IL-7 and impact on NKG2D, KIR2DL1, and KIR2DL2 expression," Experimental Hematology, vol. 38, no. 5, pp. 351-362, 2010.

[89] Y. Z. Jiang, A. J. Barrett, J. M. Goldman, and D. A. Mavroudis, "Association of natural killer cell immune recovery with a graft-versus-leukemia effect independent of graft-versus-host disease following allogeneic bone marrow transplantation," Annals of Hematology, vol. 74, no. 1, pp. 1-6, 1997. 


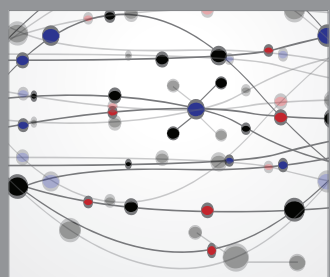

The Scientific World Journal
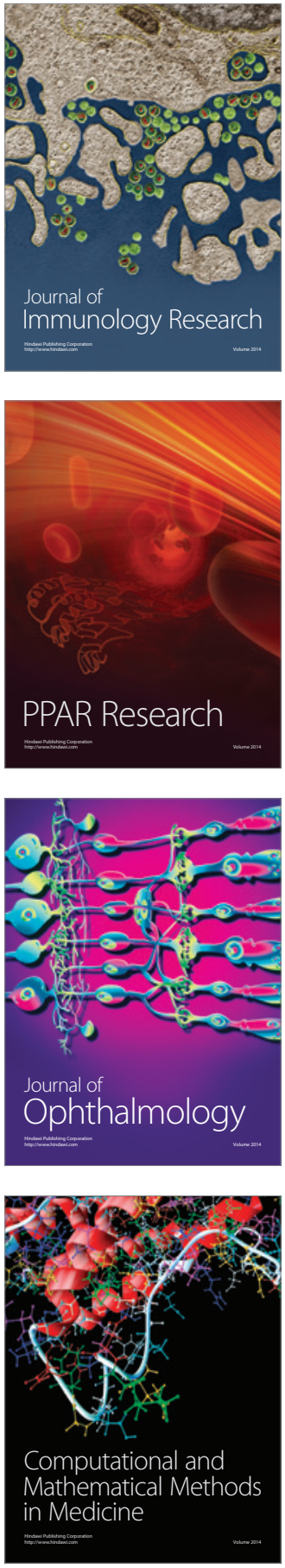

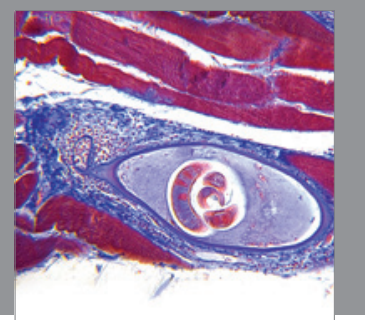

Gastroenterology

Research and Practice
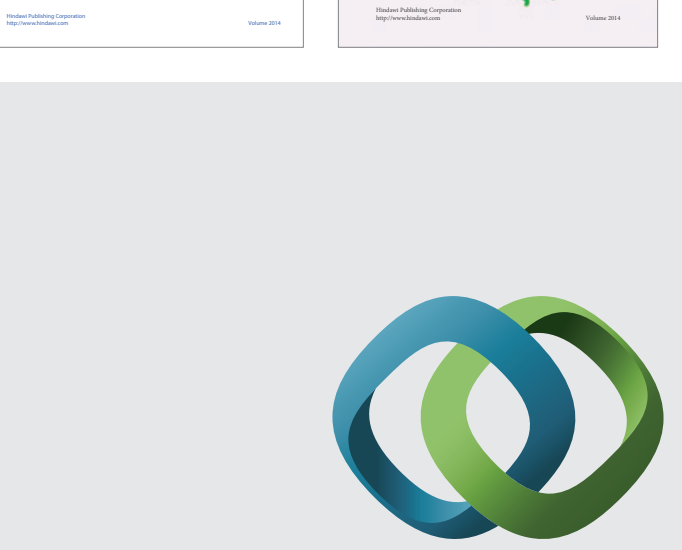

\section{Hindawi}

Submit your manuscripts at

http://www.hindawi.com
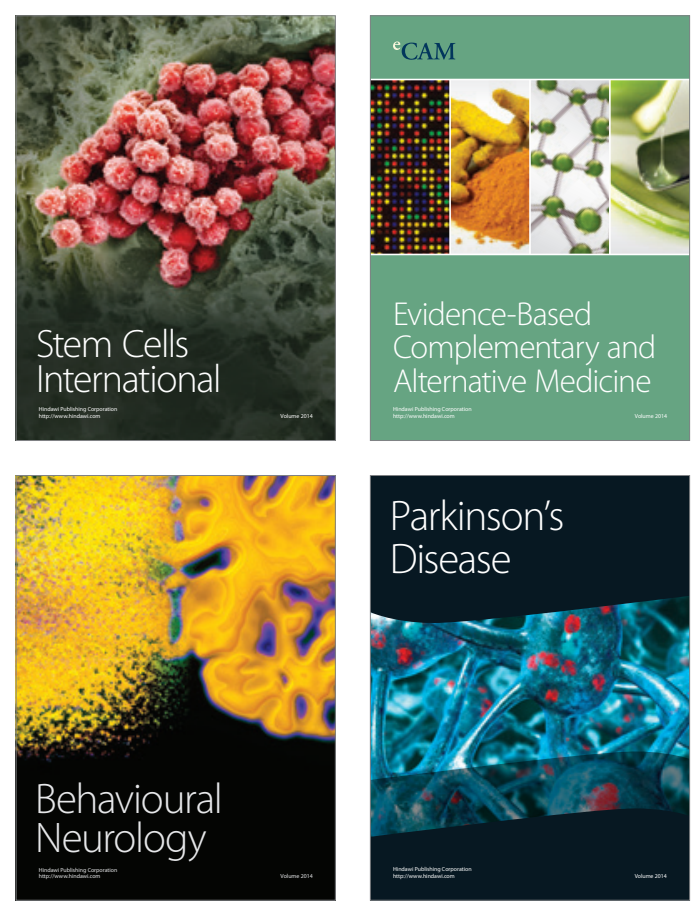

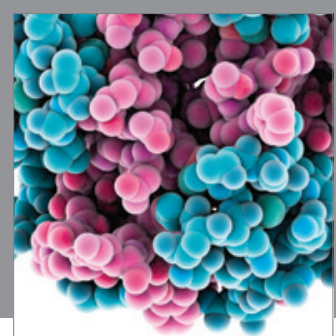

Journal of
Diabetes Research

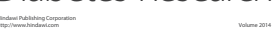

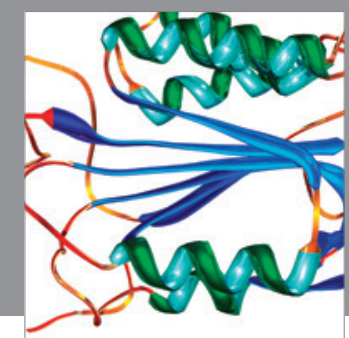

Disease Markers
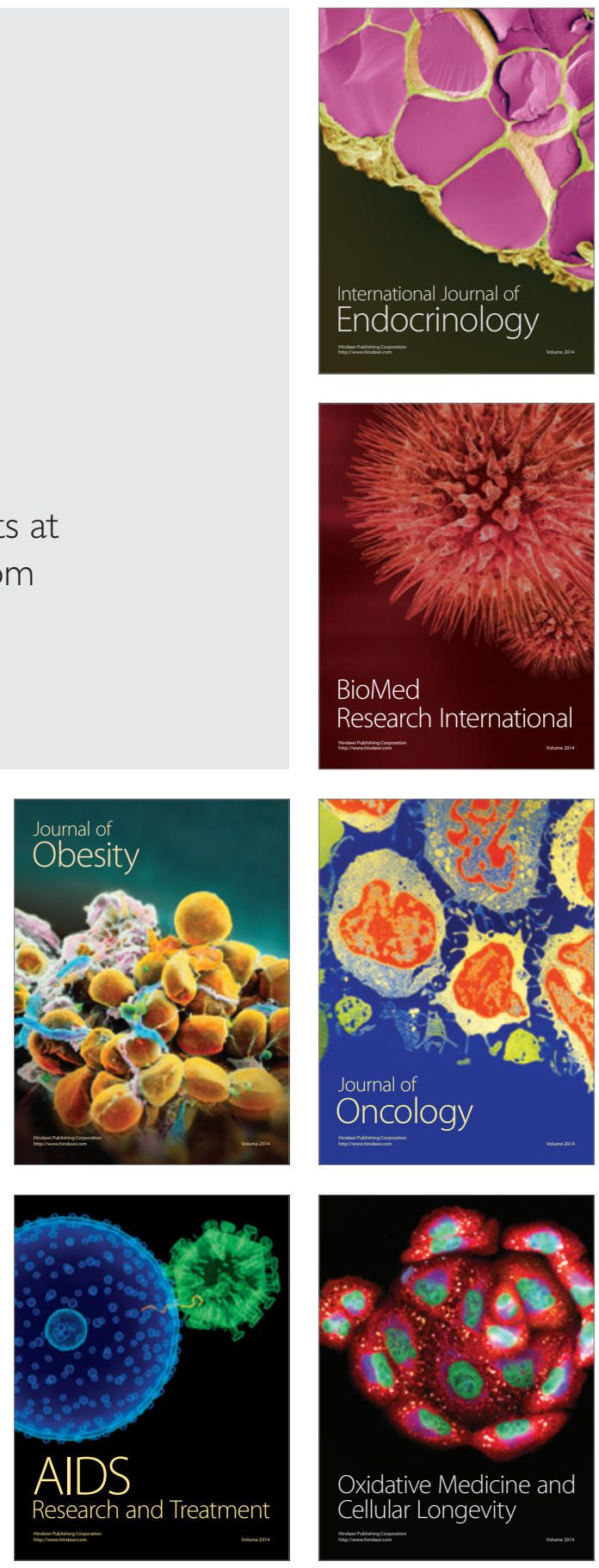